ALEA, Lat. Am. J. Probab. Math. Stat. 14, 245-247 (2017)

DOI: 10.30757/ALEA.v14-14

\title{
Erratum to: "A four moments theorem for Gamma limits on a Poisson chaos"
}

published in ALEA, Lat. Am. J. Probab. Math. Stat. 13, 163-192 (2016)

\section{Tobias Fissler and Christoph Thäle}

University of Bern

Institute of Mathematical Statistics

and Actuarial Science

Sidlerstrasse 5

CH-3012 Bern, Switzerland.

E-mail address: tobias.fissler@stat.unibe.ch

URL: http://www.imsv .unibe.ch/about_us/staff/fissler_tobias/index_eng.html

Ruhr-University Bochum

Faculty of Mathematics, NA 3/68

D-44781 Bochum, Germany.

E-mail address: christoph.thaele@rub.de

URL: http://www.ruhr-uni-bochum.de/ffm/Lehrstuehle/Thaele/christoph.html

Abstract. This note corrects a condition in Theorem 3.5 in our paper Fissler and Thäle (2016).

It has been pointed out to us that the assertions of the equivalence stated in Theorem 3.5(b) of our paper Fissler and Thäle (2016) cannot be satisfied by a sequence of kernels $f_{n} \in L_{s}^{2}\left(\mu_{n}^{q}\right)$ in the case $q=4$. Indeed, the sign condition $f_{n} \leq 0$ implies that $\mathbb{E}\left[I_{q}\left(f_{n}\right)^{3}\right] \leq 0$ in view of equation (2.8) in Fissler and Thäle (2016). On the other hand, if $I_{q}\left(f_{n}\right)$ converges in distribution to $Y \sim \bar{\Gamma}_{\nu}$, as $n \rightarrow \infty$, the uniform integrability of $\left\{I_{q}\left(f_{n}\right)^{4}: n \geq 1\right\}$ implies that

$$
\lim _{n \rightarrow \infty} \mathbb{E}\left[I_{q}\left(f_{n}\right)^{3}\right]=\mathbb{E}\left[Y^{3}\right]=8 \nu>0,
$$

which is a contradiction. In line with the equivalence, neither assertion (ii) nor (iii) can be satisfied if $f_{n} \leq 0$. E.g. $c_{q}^{2}\left\|f_{n}\right\|^{2} \leq\left\|f_{n} \widetilde{\star}_{q / 2}^{q / 2} f_{n}-c_{q} f_{n}\right\|^{2} \rightarrow 0$ for $f_{n} \leq 0$, but at the same time $q !\left\|f_{n}\right\|^{2} \rightarrow 2 \nu>0$. This contradiction also affects the results based on Theorem 3.5(b), namely Corollary 3.8(b), Theorem 4.6(b), and Theorem 4.10(b).

In Section 5.4 and, in particular, in Proposition 5.11 of Fissler and Thäle (2016), we described an alternative way to a four moments theorem in the case $q=4$ and for non-negative kernels under stronger conditions on the contraction norms of the

Received by the editors January 24th, 2017. 
kernels $f_{n}$. Against this background, Theorem 3.5 holds upon replacing condition (b) there by

(b') $q=4, f_{n} \geq 0$ for all $n \geq 1$ and $\lim _{n \rightarrow \infty}\left\|f_{n} \star_{p}^{q-p} f_{n}\right\|=0$ for all $p \in\{q / 2+$ $1, \ldots, q\}$.

Mutatis mutandis, condition (b) in Corollary 3.8 should be replaced by

(b') $q=4, f_{n} \leq 0$ for all $n \geq 1$ and $\lim _{n \rightarrow \infty}\left\|f_{n} \star_{p}^{q-p} f_{n}\right\|=0$ for all $p \in\{q / 2+$ $1, \ldots, q\}$

condition (b) in Theorem 4.6 should be replaced by

(b') $q=4, f_{n} \geq 0$ for all $n \geq 1$ and $\lim _{n \rightarrow \infty}\left\|f_{n} \star_{p}^{q-p} f_{n}\right\|=0$ for all $p \in\{q / 2+$ $1, \ldots, q\}$

and condition (b) in Theorem 4.10 should be replaced by

(b') $h_{n} \geq 0$ for all $n \geq 1$ and $\lim _{n \rightarrow \infty}\left\|h_{n} \star_{p}^{q-p} h_{n}\right\|=0$ for all $p \in\{q / 2+1, \ldots, q\}$ if $q=4$.

We remark that also under the new condition (b') in Theorem 3.5, all the technical lemmas established in Fissler and Thäle (2016, Section 5) are needed to prove the result.

We finally remark that in Fissler and Thäle (2016, Lemma 5.7), we assumed that the kernels $f_{n}$ have constant sign, i.e., that either $f_{n} \leq 0$ or $f_{n} \geq 0$. However, for $q=2$, one can dispense with the sign condition. Indeed, using the notation from Fissler and Thäle (2016), we have that

$$
\begin{aligned}
A^{\prime}\left(I_{2}\left(f_{n}\right)\right) & =\left\|G_{1}^{2} f_{n}\right\|^{2}+6\left\|G_{3}^{2} f_{n}\right\|^{2}+8\left\|f_{n} \widetilde{\star}_{2}^{0} f_{n}\right\|^{2}+48\left\|f_{n} \widetilde{\star}_{1}^{1} f_{n}-f_{n}\right\|^{2} \\
& =16\left\|f_{n} \star_{2}^{1} f_{n}\right\|^{2}+96\left\|f_{n} \widetilde{\star}_{1}^{0} f_{n}\right\|^{2}+8\left\|f_{n}^{2}\right\|^{2}+48\left\|f_{n} \widetilde{\star}_{1}^{1} f_{n}-f_{n}\right\|^{2} .
\end{aligned}
$$

Hence, assertions (1) and (2) follow directly. This is of importance because in Lemma 5.9(a) we imposed no sign condition, but referred to Lemma 5.7.

Theorem 1.6 of the recent paper Döbler and Peccati (2017) is very close to establishing a four moments theorem for Poisson integrals with a Gamma limit. However, as discussed in Remark 1.7 ibidem, one sufficient condition which implies the four moments theorem is that certain contraction norms of the kernels converge to zero in the $L^{2}$-sense. This corresponds to our additional assumption (b') from above. However, the theory developed in Döbler and Peccati (2017) allows to remove our restrictive condition on the order of the integrals as well as the sign condition on the kernels $f_{n}$.

\section{Acknowledgements}

We would like to thank Giovanni Peccati and Christian Döbler for pointing out the contradiction to us.

\section{References}

C. Döbler and G. Peccati. The fourth moment theorem on the Poisson space. ArXiv Mathematics e-prints (2017). arXiv: 1701.03120. 
T. Fissler and C. Thäle. A four moments theorem for Gamma limits on a Poisson chaos. ALEA Lat. Am. J. Probab. Math. Stat. 13 (1), 163-192 (2016). MR3476211. 